\title{
SUPPLEMENTARY MATERIAL FOR "A RATE OPTIMAL PROCEDURE FOR RECOVERING SPARSE DIFFERENCES BETWEEN HIGH-DIMENSIONAL MEANS UNDER DEPENDENCE"
}

\author{
By Jun Li* AND Ping-Shou Zhong ${ }^{\dagger}$ \\ Kent State University* and Michigan State University ${ }^{\dagger}$ \\ This supplement document provides the proofs of Lemmas 1-4 \\ and Theorems 1-5 for the main paper by Li and Zhong (2015).
}

\section{A.1. Proofs of Lemmas 1-2.}

Lemma 1. For any positive definite matrix $A_{p, p}=\left(a_{i j}\right)_{p \times p}$ and its inverse $B_{p, p}=\left(b_{i j}\right)_{p \times p}$, the following inequality holds

$$
a_{i i} \cdot b_{i i} \geq 1 \quad i=1, \cdots, p
$$

Proof. We first show that $a_{p p} \cdot b_{p p} \geq 1$. To this end, we write

$$
A_{p, p}=\left(\begin{array}{cc}
A_{p-1, p-1} & a_{p-1,1} \\
a_{p-1,1}^{T} & a_{p p}
\end{array}\right) .
$$

Then using the result from matrix inversion in block form, we have

$$
b_{p p}=\left(a_{p p}-a_{p-1,1}^{T} A_{p-1, p-1}^{T} a_{p-1,1}\right)^{-1},
$$

which implies that $a_{p p} \cdot b_{p p} \geq 1$ since $a_{p-1,1}^{T} A_{p-1, p-1}^{T} a_{p-1,1} \geq 0$.

For any $i$, we can switch $a_{i i}$ from its original position to the position $(p, p)$ using the permutation matrix $P_{p, p}$. Accordingly, $b_{i i}$ is moved from its original location to $(p, p)$ by the same matrix $P_{p, p}$. By the fact that the permutation matrix is also the orthogonal matrix, we have

$$
P_{p, p} A_{p, p} P_{p, p} P_{p, p} B_{p, p} P_{p, p}=I_{p, p}
$$

Therefore, from (A.1), we have $a_{i i} \cdot b_{i i} \geq 1$ for any $i$. This completes the proof of Lemma 1.

For any $k \in\{1, \cdots, p\}$, we let

$$
A_{k}(\Omega)=\left\{l: 1 \leq l \leq p,\left|\omega_{k l}\right| \geq L_{p}^{-1}\right\}
$$


and $B_{k}$ be the event that $\left\{\delta_{l}=0\right.$ for all $l \neq k$ and $\left.l \in A_{k}(\Omega)\right\}$. If $\Omega$ is unknown, it can be estimated by $\hat{\Omega}$ [Cai, Liu and Luo (2011)] such that with probability $1-O\left(p^{-\tau}\right)$ where $\tau$ is a positive constant,

$$
\|\hat{\Omega}-\Omega\|_{L_{1}}=O_{p}\left\{c_{p} M_{p}^{1-q}\left(\frac{\log p}{n}\right)^{\frac{1-q}{2}}\right\} .
$$

Then, let $D_{p}$ be the event $\left\{\|\hat{\Omega}-\Omega\|_{L_{1}} \leq c_{p} M_{p}^{1-q}\left(\frac{\log p}{n}\right)^{\frac{1-q}{2}}\right\}$.

Lemma 2. Assume conditions (C1), (C3) and (C4). For $\beta \in(1 / 2,1)$ and over the event $\left\{\delta_{k} \neq 0\right\} \cap B_{k} \cap D_{p}$,

$$
\hat{\delta}_{\Omega, k}=\omega_{k k} \delta_{k}+o\left\{(\log p / n)^{1 / 2}\right\} .
$$

Proof: We first consider that $\Omega$ is known. By condition (C3), the number of elements in set $A_{k}(\Omega)$ satisfies that $\left|A_{k}(\Omega)\right| \leq M_{p} L_{p}$. Since $\beta>1 / 2$, conditions (C1) and (C3) lead to

$$
\mathrm{P}\left(\delta_{k} \neq 0, B_{k}^{c}\right) \leq \sum_{l \in A_{k}, l \neq k} \mathrm{P}\left(\delta_{k} \neq 0, \delta_{l} \neq 0\right) \leq M_{p} L_{p} p^{-2 \beta}=o\left(p^{-1}\right) .
$$

Note that $\delta_{\Omega, k}=\sum_{l \in A_{k}} \omega_{k l} \delta_{l}+\sum_{l \in A_{k}^{c}} \omega_{k l} \delta_{l}$. Over the event $\left\{\delta_{k} \neq 0\right\} \cap B_{k}$, $\sum_{l \in A_{k}} \omega_{k l} \delta_{l}=\omega_{k k} \delta_{k}$. Moreover, for $l \in A_{k}^{c},\left|\omega_{k l}\right|^{q-1} / L_{p}^{1-q}>1$. Therefore, using condition (C3) again, we have

$$
\left|\delta_{\Omega, k}-\omega_{k k} \delta_{k}\right| \leq \max _{l}\left|\delta_{l}\right| L_{p}^{q-1} \sum_{l \in A_{k}^{c}}\left|\omega_{k l}\right|^{q} \leq \max _{l}\left|\delta_{l}\right| L_{p}^{q-1} c_{p} .
$$

Since $\left|\delta_{l}\right| \sim \sqrt{2 r \log p / n}$ and $q<1$, we can choose a large enough slowly varying function $L_{p}$ such that $\max _{l}\left|\delta_{l}\right| L_{p}^{q-1} c_{p}=o\left\{(\log p / n)^{1 / 2}\right\}$. Thus, we have $\delta_{\Omega, k}=\omega_{k k} \delta_{k}+o\left\{(\log p / n)^{1 / 2}\right\}$.

Next, we consider that $\Omega$ is unknown. If $\tau>1, \mathrm{P}\left(D_{p}^{c}\right)=o\left(p^{-1}\right)$ by the definition of the event of $D_{p}$. Note that $\hat{\delta}_{\Omega, k}=\delta_{\Omega, k}+\{(\hat{\Omega}-\Omega) \delta\}_{k}$. Then over the event $D_{p}$ and by condition (C4),

$$
\{(\hat{\Omega}-\Omega) \delta\}_{k} \leq \max _{l}\left|\delta_{l}\right| \cdot \| \hat{\Omega}-\left.\Omega\right|_{L_{1}}=o\left\{(\log p / n)^{1 / 2}\right\}
$$

Then, over the event $\left\{\delta_{k} \neq 0\right\} \cap B_{k} \cap D_{p}, \hat{\delta}_{\Omega, k}=\omega_{k k} \delta_{k}+o\left\{(\log p / n)^{1 / 2}\right\}$. This completes the proof of Lemma 2 . 
A.2. Proofs of Lemma 3. Recall that in Lemma $2, B_{k}$ is the event that $\left\{\delta_{l}=0\right.$ for all $l \neq k$ and $\left.l \in A_{k}\right\}$. Since $\underline{\omega} r<(\sqrt{1-\Lambda}+\sqrt{1-\beta})^{2}$, it can be shown that $\left\{(\underline{\omega} r-\beta+\Lambda)^{2}+4 \underline{\omega} r \beta\right\} /(4 \underline{\omega} r)<1$. Then using (A.2), we know that it is sufficient to prove Lemma 3 over the event $B_{k}$. Without loss of generality, we assume $\sqrt{n} \delta_{k}=\sqrt{2 r \log p}$. The result for $\sqrt{n} \delta_{k}<\sqrt{2 r \log p}$ and negative signals can be derived similarly. Note that $s<(\underline{\omega} r+\beta-\Lambda)^{2} /(4 \underline{\omega} r)=$ $\left\{(\underline{\omega} r+\beta-\Lambda)^{2} /(2 \underline{\omega} r)^{2}\right\}(\underline{\omega} r)<\underline{\omega} r$ since $\underline{\omega} r>\beta-\Lambda$. Then, over the event $B_{k}$

$$
\begin{aligned}
& \mathrm{P}\left(T_{k}<2 s \log p, \delta_{k} \neq 0\right) \\
\leq & \mathrm{P}\left(\delta_{k} \neq 0\right) \mathrm{P}\left(T_{k}<2 s \log p \mid \delta_{k} \neq 0 \cap B_{k}\right) \\
= & p^{-\beta}\left\{\mathrm{P}\left(\frac{\sqrt{n}\left(\bar{Z}_{1}^{(k)}-\bar{Z}_{2}^{(k)}\right)}{\sqrt{\omega_{k k}}}-\frac{\sqrt{n} \delta_{\Omega_{k}}}{\sqrt{\omega_{k k}}}<\sqrt{2 s \log p}-\frac{\sqrt{n} \delta_{\Omega_{k}}}{\sqrt{\omega_{k k}}} \mid \delta_{k} \neq 0 \cap B_{k}\right)\right. \\
& \left.-\mathrm{P}\left(\frac{\sqrt{n}\left(\bar{Z}_{1}^{(k)}-\bar{Z}_{2}^{(k)}\right)}{\sqrt{\omega_{k k}}}-\frac{\sqrt{n} \delta_{\Omega_{k}}}{\sqrt{\omega_{k k}}}<-\sqrt{2 s \log p}-\frac{\sqrt{n} \delta_{\Omega_{k}}}{\sqrt{\omega_{k k}}} \mid \delta_{k} \neq 0 \cap B_{k}\right)\right\} \\
\leq & p^{-\beta} L_{p} p^{-\left(\sqrt{\omega_{k k}}-\sqrt{s}\right)^{2}}\{1+o(1)\} \\
\leq & p^{-\beta} L_{p} p^{-(\sqrt{\underline{\omega}}-\sqrt{s})^{2}}\{1+o(1)\} .
\end{aligned}
$$

Since $s<(\underline{\omega} r+\beta-\Lambda)^{2} /(4 \underline{\omega} r)$, we have

$$
\sum_{k=1}^{p} \mathrm{P}\left(T_{k}<2 s \log p, \delta_{k} \neq 0\right) \leq L_{p} p^{1-\left\{(\underline{\omega} r-\beta+\Lambda)^{2}+4 \underline{\omega} r \beta\right\} /(4 \underline{\omega} r) .}
$$

Next we consider that $\Omega$ is unknown. Let $D_{p}^{*}$ be the event

$$
\begin{array}{r}
\left\{\max _{1 \leq k \leq p}\left|\sum_{l}\left(\hat{\Omega}_{k l}-\Omega_{k l}\right)\left(\bar{X}_{1}^{(l)}-\bar{X}_{2}^{(l)}\right)\right| \leq c_{p} M_{p}^{1-q}\left(\frac{\log p}{n}\right)^{1-\frac{q}{2}}\right. \\
\left.\max _{1 \leq k \leq p}\left|\hat{\omega}_{k k}-\omega_{k k}\right| \leq c_{p} M_{p}^{1-q}\left(\frac{\log p}{n}\right)^{\frac{1-q}{2}}\right\} .
\end{array}
$$

Then it can be shown that $\mathrm{P}\left(D_{p}^{* c}\right)=o\left(p^{-1}\right)$ if $\mathrm{P}\left(D_{p}^{c}\right)=o\left(p^{-1}\right)$ where $D_{p}$ is the event $\left\{\|\hat{\Omega}-\Omega\|_{L_{1}} \leq c_{p} M_{p}^{1-q}\left(\frac{\log p}{n}\right)^{\frac{1-q}{2}}\right\}$ defined in Lemma 2 .

Note that

$$
\begin{aligned}
\left|\hat{T}_{k}^{\frac{1}{2}}\right| & =\mid\left\{\frac{\sqrt{n} \sum_{l} \Omega_{k l}\left(\bar{X}_{1}^{(l)}-\bar{X}_{2}^{(l)}\right)}{\sqrt{\omega_{k k}}}\right. \\
& \left.+\frac{\sqrt{n} \sum_{l}\left(\hat{\Omega}_{k l}-\Omega_{k l}\right)\left(\bar{X}_{1}^{(l)}-\bar{X}_{2}^{(l)}\right)}{\sqrt{\omega_{k k}}}\right\} \frac{1}{1+\frac{\sqrt{\hat{\omega}_{k k}}-\sqrt{\omega_{k k}}}{\sqrt{\omega_{k k}}}} \mid,
\end{aligned}
$$


which by condition (C4), leads to

$$
\begin{aligned}
& \mathrm{P}\left(\hat{T}_{k}<2 s \log p, \delta_{k} \neq 0\right) \\
\leq & p^{-\beta} \mathrm{P}\left(\hat{T}_{k}<2 s \log p \mid \delta_{k} \neq 0 \cap B_{k} \cap D_{p}^{*}\right)+\mathrm{P}\left(\delta_{k} \neq 0 \cap B_{k}^{c}\right)+\mathrm{P}\left(D_{p}^{* c}\right) \\
\leq & p^{-\beta} \mathrm{P}\left(T_{k}<2 s \log p \mid \delta_{k} \neq 0\right)+\mathrm{P}\left(\delta_{k} \neq 0 \cap B_{k}^{c}\right)+\mathrm{P}\left(D_{p}^{* c}\right) \\
\leq & L_{p} p^{-\left\{(\underline{\omega} r-\beta+\Lambda)^{2}+4 \underline{\omega} r \beta\right\} /(4 \underline{\omega} r)}+\mathrm{P}\left(\delta_{k} \neq 0 \cap B_{k}^{c}\right)+\mathrm{P}\left(D_{p}^{* c}\right) .
\end{aligned}
$$

Since $\mathrm{P}\left(\delta_{k} \neq 0 \cap B_{k}^{c}\right)=o\left(p^{-1}\right)$ by $(\mathrm{A} .2), \mathrm{P}\left(D_{p}^{* c}\right)=o\left(p^{-1}\right)$ and $\{(\underline{\omega} r-\beta+$ $\left.\Lambda)^{2}+4 \underline{\omega} r \beta\right\} /(4 \underline{\omega} r)<1$,

$$
\mathrm{P}\left(\hat{T}_{k}<2 s \log p, \delta_{k} \neq 0\right) \leq L_{p} p^{-\left\{(\underline{\omega} r-\beta+\Lambda)^{2}+4 \underline{\omega} r \beta\right\} /(4 \underline{\omega} r)}\{1+o(1)\} .
$$

This completes the proof of Lemma 3 .

A.3. Proofs of Lemma 4. Similar to Lemma 2.2 of Ji and Jin (2012), it can be shown that with probability $1-o\left(p^{-1}\right)$, each row of the regularized $\Omega^{*}$ defined by (4.3) in the main paper has no more than $L_{p}$ nonzero components and $\left\|\Omega-\Omega^{*}\right\|_{L_{1}} \leq c_{p} M_{p}^{1-q}(\log p / n)^{1-q}$.

Note that $\left\{(\underline{\omega} r-\beta+\Lambda)^{2}+4 \underline{\omega} r \beta\right\} /(4 \underline{\omega} r)<1$. And a connected graph with size $l+1$ for $l \geq 1$ always contains a connected graph with size $l$. Then with respect to $\left(V_{0}, \Omega^{*}\right)$, it is sufficient to show that there exists an integer $m$ such that

$$
\mathrm{P}\{\mathcal{U}(s) \text { contains a connected graph with size } m\} \leq o\left(p^{-1}\right) .
$$

Recall that $D_{p}^{*}$ is the event

$$
\begin{array}{r}
\left\{\max _{1 \leq k \leq p}\left|\sum_{l}\left(\hat{\Omega}_{k l}-\Omega_{k l}\right)\left(\bar{X}_{1}^{(l)}-\bar{X}_{2}^{(l)}\right)\right| \leq c_{p} M_{p}^{1-q}\left(\frac{\log p}{n}\right)^{1-\frac{q}{2}},\right. \\
\left.\max _{1 \leq k \leq p}\left|\hat{\omega}_{k k}-\omega_{k k}\right| \leq c_{p} M_{p}^{1-q}\left(\frac{\log p}{n}\right)^{\frac{1-q}{2}}\right\} .
\end{array}
$$

And $\mathrm{P}\left(D_{p}^{* c}\right)=o\left(p^{-1}\right)$. Therefore, it is enough to show that

$$
\mathrm{P}\left\{\mathcal{U}(s) \text { contains a connected graph with size } m, D_{p}^{*}\right\} \leq o\left(p^{-1}\right) .
$$

Since there are logarithmically large number of nonzero elements in each row or column of $\Omega^{*}$, there are at most $p L_{p}^{m}$ connected graphs with size $m$ by the result from Frieze and Molloy (1999). As a result, by the union bound, it is sufficient to show that for any connected graph of size $m$, say $\mathcal{I}=\left\{k_{1}, \cdots, k_{m}\right\}$,

$$
\mathrm{P}\left\{\mathcal{I} \subset \mathcal{U}(s), D_{p}^{*}\right\} \leq o\left(p^{-2}\right) .
$$


Let $\hat{T}=\left(\hat{T}_{1}, \cdots, \hat{T}_{p}\right)^{T}$ and $\mathbf{1}_{p}=(1, \cdots, 1)^{T}$ be a $p$-dimensional vector with all components equal to 1 . Then we need to show that

$$
\mathrm{P}\left(\hat{T}^{\mathcal{I}} \geq 2 s \log p \mathbf{1}_{p}^{\mathcal{I}}, D_{p}^{*}\right) \leq o\left(p^{-2}\right) .
$$

Since for any $1 \leq k \leq p$,

$$
\begin{aligned}
\hat{T}_{k} & =\left\{\frac{\sqrt{n} \sum_{l} \Omega_{k l}\left(\bar{X}_{1}^{(l)}-\bar{X}_{2}^{(l)}\right)}{\omega_{k k}^{1 / 2}}\right. \\
& \left.+\frac{\sqrt{n} \sum_{l}\left(\hat{\Omega}_{k l}-\Omega_{k l}\right)\left(\bar{X}_{1}^{(l)}-\bar{X}_{2}^{(l)}\right)}{\omega_{k k}^{1 / 2}}\right\}^{2}\left(\frac{1}{1+\frac{\hat{\omega}_{k k}-\omega_{k k}}{\omega_{k k}}}\right),
\end{aligned}
$$

we only need to show that

$$
\mathrm{P}\left\{\left|\left(T^{\mathcal{I}}\right)^{1 / 2}\right| \geq(2 s \log p)^{1 / 2} \mathbf{1}_{p}^{\mathcal{I}}, D_{p}^{*}\right\} \leq o\left(p^{-2}\right) .
$$

Let $\tilde{\delta}_{\Omega}^{\mathcal{I}}=\left(\delta_{\Omega, k_{1}} / \omega_{k_{1} k_{1}}^{1 / 2}, \cdots, \delta_{\Omega, k_{m}} / \omega_{k_{m} k_{m}}^{1 / 2}\right)$ and $\mathcal{E}=\left(T^{\mathcal{I}}\right)^{1 / 2}-\sqrt{n} \tilde{\delta}_{\Omega}^{\mathcal{I}}$ where $T=\left(T_{1}, \cdots, T_{p}\right)^{T}$. Then it can be shown that

$$
\mathcal{E} \sim \mathrm{N}\left(0, \bar{\Omega}^{\mathcal{I}, \mathcal{I}}\right)
$$

where $\bar{\Omega}_{i j}=\omega_{i j} /\left(\omega_{i i} \omega_{j j}\right)^{1 / 2}$ for $i, j \in \mathcal{I}$. By Cauchy-Schwartz inequality,

$$
\|\mathcal{E}\|^{2} \geq \frac{1}{2}\left\|\left(T^{\mathcal{I}}\right)^{1 / 2}\right\|^{2}-\left\|\sqrt{n} \tilde{\delta}_{\Omega}^{\mathcal{I}}\right\|^{2} .
$$

Note that the largest eigenvalue of $\bar{\Omega}^{\mathcal{I}, \mathcal{I}}$ is not greater than that of $\bar{\Omega}$, and the latter has the largest eigenvalue no greater than $C_{0} / \underline{\omega} \leq C_{0}$ since $\underline{\omega} \geq 1$. Therefore,

$$
\mathcal{E}^{T}\left(\bar{\Omega}^{\mathcal{I}, \mathcal{I}}\right)^{-1} \mathcal{E} \geq \frac{1}{C_{0}}\|\mathcal{E}\|^{2}
$$

Moreover, by the construction of the thresholding,

$$
\left\|\left(T^{\mathcal{I}}\right)^{1 / 2}\right\|^{2} \geq 2 m s \log p .
$$

Combining (A.3), (A.4) and (A.5), we have

$$
\mathcal{E}^{T}\left(\bar{\Omega}^{\mathcal{I}, \mathcal{I}}\right)^{-1} \mathcal{E} \geq \frac{1}{C_{0}}\left(m s \log p-\left\|\sqrt{n} \tilde{\delta}_{\Omega}^{\mathcal{I}}\right\|^{2}\right) .
$$

Using Lemma A.3 of Ji and Jin (2012), we can show that

$$
\mathrm{P}\left[\left\|\sqrt{n} \tilde{\delta}_{\Omega}^{\mathcal{I}}\right\|^{2} \geq\left\{C_{0} g+c m L_{p}(\log p / n)^{2(1-q)}\right\}(2 s \log p), D_{p}^{*}\right] \leq L_{p} p^{-\beta g},
$$


where $g$ is chosen to satisfy $\left\{C_{0} g+c m L_{p}(\log p / n)^{2(1-q)}\right\}(2 s \log p) \leq \frac{1}{2} m s \log p$. Denote $A_{p}$ to be the event

$$
\left\{\left\|\sqrt{n} \tilde{\delta}_{\Omega}^{\mathcal{I}}\right\|^{2} \geq\left\{C_{0} g+c m L_{p}(\log p / n)^{2(1-q)}\right\}(2 s \log p)\right\} .
$$

Then, we have $\mathrm{P}\left(A_{p} \cap D_{p}^{*}\right) \leq L_{p} p^{-\beta g}$. Similar to Lemma A.3 of Ji and Jin (2012), for sufficiently large $m$,

$\mathrm{P}\left\{\mathcal{I} \subset \mathcal{U}(s), D_{p}^{*}\right\} \leq \mathrm{P}\left\{\mathcal{E}^{T}\left(\bar{\Omega}^{\mathcal{I}, \mathcal{I}}\right)^{-1} \mathcal{E} \geq \frac{1}{2 C_{0}}(m s \log p)\right\}+\mathrm{P}\left(A_{p} \cap D_{p}^{*}\right)=o\left(p^{-2}\right)$.

This completes the proof of Lemma 4.

A.4. Proof of Theorem 1. To make the discussion easier, we change the two-sample problem into a one-sample problem. Without loss of generality, we assume $n_{1} \leq n_{2}$ and define

$$
Y_{i}=X_{1 i}-\sqrt{\frac{n_{1}}{n_{2}}} X_{2 i}+\frac{1}{\sqrt{n_{1} n_{2}}} \sum_{j=1}^{n_{1}} X_{2 j}-\frac{1}{n_{2}} \sum_{l=1}^{n_{2}} X_{2 l} \quad i=1, \cdots, n_{1}
$$

It can be shown that $Y_{i} \stackrel{i . i . d .}{\sim} \mathrm{N}\left(\delta, \Sigma_{1}+n_{1} / n_{2} \Sigma_{2}\right)$ for $i=1, \cdots, n_{1}$ under the model (1.1) in the main paper.

Note that the loss function for $j$ th dimension is $L\left(\theta_{j}, \hat{\theta}_{j}\right)=\theta_{j}\left(1-\hat{\theta}_{j}\right)+$ $p^{-\Lambda}\left(1-\theta_{j}\right) \hat{\theta}_{j}$ where $\theta_{j}=0$ if $\delta_{j}=0$ and $\theta_{j}=1$ otherwise, and $\hat{\theta}_{j}$ is the decision rule with value equal to either 0 or 1 . Since the total loss $L(\theta, \hat{\theta})=\sum_{j} L\left(\theta_{j}, \hat{\theta}_{j}\right)$, we only focus on the $j$ th loss function and derive its corresponding risk function.

Toward this end, we first derive an optimal Bayesian rule by marginalizing out $\delta_{j}$ and letting $\tilde{\delta}=\delta-\delta_{j} e_{j}$ where $e_{j}$ is a $p \times 1$ unit vector with non-zero value 1 at the $j$ th component, and the full parameterization will be used later to study the corresponding risk function. Since $\delta=\left(\delta_{1}, \cdots, \delta_{p}\right)^{T}, \tilde{\delta}$ is obtained by deleting the $j$ th component $\delta_{j}$ from $\delta$. Let $h\left(\mathcal{Y} ; \tilde{\delta}, \delta_{j}\right)$ be the joint density of $\left(Y_{1}, \cdots, Y_{n_{1}}\right)$ where $Y_{i}$ is defined in (A.6):

$$
\begin{aligned}
h\left(\mathcal{Y} ; \tilde{\delta}, \delta_{j}\right) & =(2 \pi)^{-n_{1} p / 2}|\tilde{\Sigma}|^{-n_{1} / 2} \exp \left(-\frac{1}{2} \sum_{i=1}^{n_{1}}\left\{\left(Y_{i}-\tilde{\delta}\right)^{T} \tilde{\Sigma}^{-1}\left(Y_{i}-\tilde{\delta}\right)\right\}\right) \\
& \times \exp \left\{\delta_{j} e_{j}^{T} \tilde{\Sigma}^{-1} \sum_{i=1}^{n_{1}}\left(Y_{i}-\tilde{\delta}\right)-\frac{n_{1}}{2} \delta_{j}^{2} \tilde{\omega}_{j j}\right\} \\
& =h(\mathcal{Y} ; \tilde{\delta}, 0) \exp \left\{\delta_{j} e_{j}^{T} \tilde{\Sigma}^{-1} \sum_{i=1}^{n_{1}}\left(Y_{i}-\tilde{\delta}\right)-\frac{n_{1}}{2} \delta_{j}^{2} \tilde{\omega}_{j j}\right\}
\end{aligned}
$$


where $\tilde{\Sigma}=\left(\Sigma_{1}+n_{1} / n_{2} \Sigma_{2}\right)$ and $\tilde{\omega}_{j j}$ is the $j$ th diagonal element of $\tilde{\Sigma}^{-1}$. If we let

$f_{j, 0}(\mathcal{Y})=\int h(\mathcal{Y} ; \tilde{\delta}, 0) d F(\tilde{\delta}) \quad$ and $\quad f_{j, 1}(\mathcal{Y})=\int h\left(\mathcal{Y} ; \tilde{\delta}, \delta_{j}\right) d \kappa_{p}\left(\delta_{j}\right) d F(\tilde{\delta})$,

where $F(\tilde{\delta})$ is the joint $\mathrm{CDF}$ of $\tilde{\delta}=\left(\delta_{1}, \cdots, \delta_{j-1}, 0, \delta_{j+1}, \cdots, \delta_{p}\right)^{T}$ and $\kappa_{p}\left(\delta_{j}\right)$ is the $\mathrm{CDF}$ of $\delta_{j}$ defined in condition $(\mathrm{C} 1)$, then it can be shown that the Bayesian decision rule that minimizes the risk function for the $j$ th dimension is given by (2.3) in the main paper [ $\mathrm{Li}$ and Zhong (2015)]. Based on the Bayesian rule, the risk function can be derived by following similar derivations given in Theorem 2 of [Sun and Cai (2007)], Theorem 2 of [Xie, Cai and $\mathrm{Li}$ (2011)] and Theorem 1.1 of [Ji and Jin (2012)]. More specifically, the risk function at the $j$ th dimension is

$$
\begin{aligned}
H_{j} & =\mathrm{E}\left\{L\left(\theta_{j}, \hat{\theta}_{j}\right)\right\} \\
& =\mathrm{E}\left(\mathrm{E}\left\{L\left(\theta_{j}, \hat{\theta}_{j}\right)\right\} \mid \mathcal{Y}\right) \\
& =\mathrm{E}\left\{\frac{\epsilon_{p} f_{j, 1}(\mathcal{Y})}{\left(1-\epsilon_{p}\right) f_{j, 0}(\mathcal{Y})+\epsilon_{p} f_{j, 1}(\mathcal{Y})}\left(1-\hat{\theta}_{j}\right)\right. \\
& \left.+p^{-\Lambda} \frac{\left(1-\epsilon_{p}\right) f_{j, 0}(\mathcal{Y})}{\left(1-\epsilon_{p}\right) f_{j, 0}(\mathcal{Y})+\epsilon_{p} f_{j, 1}(\mathcal{Y})} \hat{\theta}_{j}\right\} \\
& =\int \frac{\epsilon_{p} f_{j, 1}(\mathcal{Y})}{\left(1-\epsilon_{p}\right) f_{j, 0}(\mathcal{Y})+\epsilon_{p} f_{j, 1}(\mathcal{Y})}\left(1-\hat{\theta}_{j}\right)\left\{\left(1-\epsilon_{p}\right) f_{j, 0}(\mathcal{Y})+\epsilon_{p} f_{j, 1}(\mathcal{Y})\right\} d \mathcal{Y} \\
& +p^{-\Lambda} \int \frac{\left(1-\epsilon_{p}\right) f_{j, 0}(\mathcal{Y})}{\left(1-\epsilon_{p}\right) f_{j, 0}(\mathcal{Y})+\epsilon_{p} f_{j, 1}(\mathcal{Y})} \hat{\theta}_{j}\left\{\left(1-\epsilon_{p}\right) f_{j, 0}(\mathcal{Y})+\epsilon_{p} f_{j, 1}(\mathcal{Y})\right\} d \mathcal{Y} \\
& =\int_{A^{c}} \epsilon_{p} f_{j, 1}(\mathcal{Y}) d \mathcal{Y}+p^{-\Lambda} \int_{A}\left(1-\epsilon_{p}\right) f_{j, 0}(\mathcal{Y}) d \mathcal{Y} \\
& =\epsilon_{p}-\int_{A}\left|p^{-\Lambda}\left(1-\epsilon_{p}\right) f_{j, 0}(\mathcal{Y})-\epsilon_{p} f_{j, 1}(\mathcal{Y})\right| d \mathcal{Y},
\end{aligned}
$$

where the set $A=\left\{\mathcal{Y}: \hat{\theta}_{j}=1\right\}$, and from line two to line three, we have used the fact that

$$
\mathrm{E}\left(\theta_{j} \mid \mathcal{Y}\right)=\mathrm{P}\left(\theta_{j}=1 \mid \mathcal{Y}\right)=\frac{\mathrm{P}\left(\mathcal{Y} \mid \theta_{j}=1\right) \mathrm{P}\left(\theta_{j}=1\right)}{\mathrm{P}\left(\mathcal{Y} \mid \theta_{j}=0\right) \mathrm{P}\left(\theta_{j}=0\right)+\mathrm{P}\left(\mathcal{Y} \mid \theta_{j}=1\right) \mathrm{P}\left(\theta_{j}=1\right)} .
$$

Another way to write line six in (A.8) is

$$
\begin{aligned}
H_{j} & =\int_{A^{c}} \epsilon_{p} f_{j, 1}(\mathcal{Y}) d \mathcal{Y}+p^{-\Lambda} \int_{A}\left(1-\epsilon_{p}\right) f_{j, 0}(\mathcal{Y}) d \mathcal{Y} \\
& =p^{-\Lambda}\left(1-\epsilon_{p}\right)-\int_{A^{c}}\left|p^{-\Lambda}\left(1-\epsilon_{p}\right) f_{j, 0}(\mathcal{Y})-\epsilon_{p} f_{j, 1}(\mathcal{Y})\right| d \mathcal{Y} .
\end{aligned}
$$


By combining (A.8) and (A.9), the risk function at the $j$ th dimension is

$$
H_{j}=\frac{1}{2}\left\{p^{-\Lambda}\left(1-\epsilon_{p}\right)+\epsilon_{p}-\int\left|p^{-\Lambda}\left(1-\epsilon_{p}\right) f_{j, 0}-\epsilon_{p} f_{j, 1}\right| d \mathcal{Y}\right\}
$$

To evaluate the above risk function, we need to evaluate both $f_{j, 0}$ and $f_{j, 1}$ given by (A.7), where integrations are taken over with respect to unspecified distributions of $\delta_{j}$ and $\tilde{\delta}$. Recall that our goal is to obtain the lower bound of $H_{j}$. Similar to the proof of Theorem 1.1 in [Ji and Jin (2012)], we first switch the order of integrations in (A.10) by applying the Fubini's Theorem:

$$
\begin{aligned}
& \int\left|p^{-\Lambda}\left(1-\epsilon_{p}\right) f_{j, 0}-\epsilon_{p} f_{j, 1}\right| d \mathcal{Y} \\
= & \int\left|\int\left\{p^{-\Lambda}\left(1-\epsilon_{p}\right) h(\mathcal{Y} ; \tilde{\delta}, 0)-\epsilon_{p} h\left(\mathcal{Y} ; \tilde{\delta}, \delta_{j}\right)\right\} d \kappa_{p}\left(\delta_{j}\right) d F(\tilde{\delta})\right| d \mathcal{Y} \\
\leq & \int Q\left(\tilde{\delta}, \delta_{j}\right) d \kappa_{p}\left(\delta_{j}\right) d F(\tilde{\delta}),
\end{aligned}
$$

where

$$
Q\left(\tilde{\delta}, \delta_{j}\right)=\int\left|p^{-\Lambda}\left(1-\epsilon_{p}\right) h(\mathcal{Y} ; \tilde{\delta}, 0)-\epsilon_{p} h\left(\mathcal{Y} ; \tilde{\delta}, \delta_{j}\right)\right| d \mathcal{Y}
$$

Then from (A.10), the lower bound of the risk function at the $j$ th dimension is

$$
H_{j} \geq \frac{1}{2}\left\{p^{-\Lambda}\left(1-\epsilon_{p}\right)+\epsilon_{p}-\int Q\left(\tilde{\delta}, \delta_{j}\right) d \kappa_{p}\left(\delta_{j}\right) d F(\tilde{\delta})\right\}
$$

which requires to evaluate $Q\left(\tilde{\delta}, \delta_{j}\right)$ given by (A.11). As we will show as follows, $Q\left(\tilde{\delta}, \delta_{j}\right)$ has two important properties: $Q\left(\tilde{\delta}, \delta_{j}\right)=Q\left(\tilde{\delta},-\delta_{j}\right)$ and $Q\left(\tilde{\delta}, \delta_{j}\right)$ is an increasing function of $\delta_{j}>0$. Toward this end, if we let $D_{p}=$ $\left\{\mathcal{Y}: \epsilon_{p} \exp \left\{\delta_{j} e_{j}^{T} \tilde{\Sigma}^{-1} \sum_{i=1}^{n_{1}}\left(Y_{i}-\tilde{\delta}\right)-n_{1} / 2 \delta_{j}^{2} \tilde{\omega}_{j j}\right\}>p^{-\Lambda}\left(1-\epsilon_{p}\right)\right\}$, then

$$
\begin{aligned}
Q\left(\tilde{\delta}, \delta_{j}\right) & =-\int_{D_{p}}\left\{p^{-\Lambda}\left(1-\epsilon_{p}\right) h(\mathcal{Y} ; \tilde{\delta}, 0)-\epsilon_{p} h\left(\mathcal{Y} ; \tilde{\delta}, \delta_{j}\right)\right\} d \mathcal{Y} \\
& +\int_{D_{p}^{c}}\left\{p^{-\Lambda}\left(1-\epsilon_{p}\right) h(\mathcal{Y} ; \tilde{\delta}, 0)-\epsilon_{p} h\left(\mathcal{Y} ; \tilde{\delta}, \delta_{j}\right)\right\} d \mathcal{Y}
\end{aligned}
$$

This, together with the fact that

$$
\begin{aligned}
p^{-\Lambda}\left(1-\epsilon_{p}\right)+\epsilon_{p} & =\int_{D_{p}}\left\{p^{-\Lambda}\left(1-\epsilon_{p}\right) h(\mathcal{Y} ; \tilde{\delta}, 0)+\epsilon_{p} h\left(\mathcal{Y} ; \tilde{\delta}, \delta_{j}\right)\right\} d \mathcal{Y} \\
& +\int_{D_{p}^{c}}\left\{p^{-\Lambda}\left(1-\epsilon_{p}\right) h(\mathcal{Y} ; \tilde{\delta}, 0)+\epsilon_{p} h\left(\mathcal{Y} ; \tilde{\delta}, \delta_{j}\right)\right\} d \mathcal{Y}
\end{aligned}
$$


leads to

$$
\begin{aligned}
Q\left(\tilde{\delta}, \delta_{j}\right) & =p^{-\Lambda}\left(1-\epsilon_{p}\right)+\epsilon_{p}-2\left\{p^{-\Lambda}\left(1-\epsilon_{p}\right) \int_{D_{p}} h(\mathcal{Y} ; \tilde{\delta}, 0) d \mathcal{Y}\right. \\
& \left.+\epsilon_{p} \int_{D_{p}^{c}} h\left(\mathcal{Y} ; \tilde{\delta}, \delta_{j}\right) d \mathcal{Y}\right\} .
\end{aligned}
$$

Define $W_{j}(\tilde{\delta})=e_{j}^{T} \tilde{\Sigma}^{-1} \sum_{i=1}^{n_{1}}\left(Y_{i}-\tilde{\delta}\right)$. Under $H_{0 j}, W_{j}(\tilde{\delta}) \sim \mathrm{N}\left(0, n_{1} \tilde{\omega}_{j j}\right)$ since $\delta_{j}=0$, and under $H_{1 j}, W_{j}(\tilde{\delta}) \sim \mathrm{N}\left(n_{1} \delta_{j} \tilde{\omega}_{j j}, n_{1} \tilde{\omega}_{j j}\right)$. Note that $D_{p}=\{\mathcal{Y}:$ $e_{j}^{T} \tilde{\Sigma}^{-1} \sum_{i=1}^{n_{1}}\left(Y_{i}-\tilde{\delta}\right)>1 / \delta_{j}\left\{\log p^{-\Lambda}+\log \left(\frac{1-\epsilon_{p}}{\epsilon_{p}}\right)+\frac{n_{1}}{2} \delta_{j}^{2} \tilde{\omega}_{j j}\right\}$ if $\delta_{j}>0$ and $D_{p}=\left\{\mathcal{Y}: e_{j}^{T} \tilde{\Sigma}^{-1} \sum_{i=1}^{n_{1}}\left(Y_{i}-\tilde{\delta}\right)<1 / \delta_{j}\left\{\log p^{-\Lambda}+\log \left(\frac{1-\epsilon_{p}}{\epsilon_{p}}\right)+\frac{n_{1}}{2} \delta_{j}^{2} \tilde{\omega}_{j j}\right\}\right.$ if $\delta_{j}<0$. Then, for $\delta_{j}>0$,

$$
\begin{aligned}
Q\left(\tilde{\delta}, \delta_{j}\right) & =p^{-\Lambda}\left(1-\epsilon_{p}\right)+\epsilon_{p}-2\left\{p^{-\Lambda}\left(1-\epsilon_{p}\right) \bar{\Phi}\left(\frac{\lambda_{p}\left(\delta_{j}\right)}{\sqrt{n_{1} \tilde{\omega}_{j j}}}\right)\right. \\
& \left.+\epsilon_{p} \Phi\left(\frac{\lambda_{p}\left(\delta_{j}\right)-n_{1} \delta_{j} \tilde{\omega}_{j j}}{\sqrt{n_{1} \tilde{\omega}_{j j}}}\right)\right\}, \\
Q\left(\tilde{\delta},-\delta_{j}\right) & =p^{-\Lambda}\left(1-\epsilon_{p}\right)+\epsilon_{p}-2\left\{p^{-\Lambda}\left(1-\epsilon_{p}\right) \Phi\left(\frac{-\lambda_{p}\left(\delta_{j}\right)}{\sqrt{n_{1} \tilde{\omega}_{j j}}}\right)\right. \\
& \left.+\epsilon_{p} \bar{\Phi}\left(\frac{-\lambda_{p}\left(\delta_{j}\right)+n_{1} \delta_{j} \tilde{\omega}_{j j}}{\sqrt{n_{1} \tilde{\omega}_{j j}}}\right)\right\},
\end{aligned}
$$

where $\lambda_{p}\left(\delta_{j}\right)=1 / \delta_{j}\left\{\log p^{-\Lambda}+\log \left(\frac{1-\epsilon_{p}}{\epsilon_{p}}\right)+\frac{n_{1}}{2} \delta_{j}^{2} \tilde{\omega}_{j j}\right\}$. Based on the property of $\Phi(\cdot)$, it follows that $Q\left(\tilde{\delta}, \delta_{j}\right)=Q\left(\tilde{\delta},-\delta_{j}\right)$.

Next we want to show that $Q\left(\tilde{\delta}, \delta_{j}\right)$ is an increasing function of $\delta_{j}>0$. Toward this end, we take the derivative of (A.13) with respect to $\delta_{j}$ and have

$$
\begin{aligned}
Q^{T}\left(\tilde{\delta}, \delta_{j}\right) & =2 p^{-\Lambda}\left(1-\epsilon_{p}\right) \phi\left(\frac{\lambda_{p}\left(\delta_{j}\right)}{\sqrt{n_{1} \tilde{\omega}_{j j}}}\right) \frac{-\frac{1}{\delta_{j}^{2}}\left\{\log p^{-\Lambda}+\log \left(\frac{1-\epsilon_{p}}{\epsilon_{p}}\right)\right\}+\frac{n_{1}}{2} \tilde{\omega}_{j j}}{\sqrt{n_{1} \tilde{\omega}_{j j}}} \\
& -2 \epsilon_{p} \phi\left(\frac{\lambda_{p}\left(\delta_{j}\right)-n_{1} \delta_{j} \tilde{\omega}_{j j}}{\sqrt{n_{1} \tilde{\omega}_{j j}}}\right) \frac{-\frac{1}{\delta_{j}^{2}}\left\{\log p^{-\Lambda}+\log \left(\frac{1-\epsilon_{p}}{\epsilon_{p}}\right)\right\}-\frac{n_{1}}{2} \tilde{\omega}_{j j}}{\sqrt{n_{1} \tilde{\omega}_{j j}}} \\
& =2 p^{-\Lambda}\left(1-\epsilon_{p}\right) \phi\left(\frac{\lambda_{p}\left(\delta_{j}\right)}{\sqrt{n_{1} \tilde{\omega}_{j j}}}\right) \sqrt{n_{1} \tilde{\omega}_{j j}}>0,
\end{aligned}
$$

where $\phi(\cdot)$ is the pdf of the standard normal distribution and we have applied the fact that

$$
\epsilon_{p} \phi\left(\frac{\lambda_{p}\left(\delta_{j}\right)-n_{1} \delta_{j} \tilde{\omega}_{j j}}{\sqrt{n_{1} \tilde{\omega}_{j j}}}\right)=p^{-\Lambda}\left(1-\epsilon_{p}\right) \phi\left(\frac{\lambda_{p}\left(\delta_{j}\right)}{\sqrt{n_{1} \tilde{\omega}_{j j}}}\right) .
$$


Hence, for $\delta_{j} \in\left[-\tau_{p}, 0\right) \cup\left(0, \tau_{p}\right]$ where $\tau_{p}=\sqrt{2 r \log p / n}$, we have $Q\left(\tilde{\delta}, \delta_{j}\right) \leq$ $Q\left(\tilde{\delta}, \tau_{p}\right)$, which, combining (A.12) and (A.13), leads to

$$
H_{j} \geq p^{-\Lambda}\left(1-\epsilon_{p}\right) \bar{\Phi}\left(\frac{\lambda_{p}\left(\tau_{p}\right)}{\sqrt{n_{1} \tilde{\omega}_{j j}}}\right)+\epsilon_{p} \Phi\left(\frac{\lambda_{p}\left(\tau_{p}\right)-n_{1} \tau_{p} \tilde{\omega}_{j j}}{\sqrt{n_{1} \tilde{\omega}_{j j}}}\right)
$$

Using $\epsilon_{p}=p^{-\beta}, \tau_{p}^{2}=2 r \log p / n$ and $n_{1} \tilde{\omega}_{j j}=n \omega_{j j}$ where $\omega_{j j}$ is the $j$ th diagonal element of $\Omega$, we have

$$
\frac{\lambda_{p}\left(\tau_{p}\right)}{\sqrt{n_{1} \tilde{\omega}_{j j}}}=\left(\frac{\beta-\Lambda}{\sqrt{2 r \omega_{j j}}}+\frac{\sqrt{r \omega_{j j}}}{\sqrt{2}}\right) \sqrt{\log p}
$$

and

$$
\frac{\lambda_{p}\left(\tau_{p}\right)-n_{1} \tau_{p} \tilde{\omega}_{j j}}{\sqrt{n_{1} \tilde{\omega}_{j j}}}=\left(\frac{\beta-\Lambda-r \omega_{j j}}{\sqrt{2 r \omega_{j j}}}\right) \sqrt{\log p} .
$$

First, if $\beta-r \omega_{j j}<\Lambda<\beta+r \omega_{j j}$, then (A.14) becomes

$$
\begin{aligned}
H_{j} & \geq p^{-\Lambda} L_{p} p^{-\frac{\left(r \omega_{j j}+\beta-\Lambda\right)^{2}}{4 r \omega_{j j}}}+p^{-\beta} L_{p} p^{-\frac{\left(r \omega_{j j}-\beta+\Lambda\right)^{2}}{4 r \omega_{j j}}} \\
& =p^{-\beta} L_{p} p^{-\frac{\left(r \omega_{j j}-\beta+\Lambda\right)^{2}}{4 r \omega_{j j}}} .
\end{aligned}
$$

Next, we consider $\Lambda<\beta-r \omega_{j j}$, then (A.14) becomes

$$
\begin{aligned}
H_{j} & \geq p^{-\Lambda} L_{p} p^{-\frac{\left(r \omega_{j j}+\beta-\Lambda\right)^{2}}{4 r \omega_{j j}}}+p^{-\beta} \\
& =p^{-\beta}\{1+o(1)\}
\end{aligned}
$$

Last, if $\Lambda>\beta+r \omega_{j j}$, then (A.14) becomes

$$
\begin{aligned}
H_{j} & \geq p^{-\Lambda}+p^{-\beta} L_{p} p^{-\frac{\left(r \omega_{j j}-\beta+\Lambda\right)^{2}}{4 r \omega_{j j}}} \\
& =p^{-\Lambda}\{1+o(1)\}
\end{aligned}
$$

Recall that $H=\sum_{j=1}^{p} H_{j}$. Using the fact that $\underline{\omega} \leq \omega_{j j} \leq \bar{\omega}$ and $\left(r \omega_{j j}+\right.$ $\beta-\Lambda)^{2} /\left(4 r \omega_{j j}\right)$ is an increasing function of $\omega_{j j}$, Theorem 1 can be derived based on the results given in (A.15), (A.16) and (A.17).

A.5. Proof of Theorem 2. Recall that in the proof of Theorem 1, we have defined the loss function $L(\theta, \hat{\theta})=\sum_{i=1}^{p}\left\{\theta_{i}\left(1-\hat{\theta}_{i}\right)+p^{-\Lambda}\left(1-\theta_{i}\right) \hat{\theta}_{i}\right\}$. For any decision rule $\hat{\theta}_{i}$, the marginal false discovery rate

$$
\mathrm{mFDR}=\frac{\mathrm{E}\left\{\sum_{i}\left(1-\theta_{i}\right) \hat{\theta}_{i}\right\}}{\mathrm{E}\left(\sum_{i} \hat{\theta}_{i}\right)}=1-\frac{\mathrm{E}\left(\sum_{i} \theta_{i} \hat{\theta}_{i}\right)}{\mathrm{E}\left(\sum_{i} \hat{\theta}_{i}\right)} .
$$


Following the similar derivation in the proof of Theorem 2.3 in Ji and Zhao (2014), we have $\mathrm{mFDR}=1+o(1)$ if $p^{1-\beta}=o\left\{\mathrm{E}\left(\sum_{i} \hat{\theta}_{i}\right)\right\}$ since $\mathrm{E}\left(\sum_{i} \theta_{i} \hat{\theta}_{i}\right) \leq$ $\min \left\{p^{1-\beta}, \mathrm{E}\left(\sum_{i} \hat{\theta}_{i}\right)\right\}$. Hence, if $\mathrm{mFDR}$ is controlled at a level $\alpha<1$, we must have either $p^{1-\beta} \sim \mathrm{E}\left(\sum_{i} \hat{\theta}_{i}\right)$ or $\mathrm{E}\left(\sum_{i} \hat{\theta}_{i}\right)=o\left(p^{1-\beta}\right)$. For the latter,

$$
\operatorname{mFNR}=\frac{\mathrm{E}\left\{\sum_{i} \theta_{i}\left(1-\hat{\theta}_{i}\right)\right\}}{\mathrm{E}\left\{\sum_{i}\left(1-\hat{\theta}_{i}\right)\right\}}=\frac{p^{1-\beta}\{1+o(1)\}}{p\{1+o(1)\}}=p^{-\beta}\{1+o(1)\} .
$$

Next, we consider the mFNR under the constraint $p^{1-\beta} \sim \mathrm{E}\left(\sum_{i} \hat{\theta}_{i}\right)$. Toward this end, we first note that if $\mathrm{mFDR} \leq \alpha<1$, the following result can be derived from (A.18):

$$
p^{-\beta} \alpha \sum_{i=1}^{p} \mathrm{E}\left(\hat{\theta}_{i} \mid \theta_{i}=1\right) \geq(1-\alpha) \sum_{i=1}^{p} \mathrm{E}\left(\hat{\theta}_{i} \mid \theta_{i}=0\right) .
$$

Recall that in the proof of Theorem 1, the optimal decision rule

$$
\hat{\theta}_{i}=\mathrm{I}\left\{\frac{\left(1-p^{-\beta}\right) f_{j, 0}(\mathcal{Y})}{p^{-\beta} f_{j, 1}(\mathcal{Y})} \leq p^{\Lambda}\right\}
$$

where $f_{j, 0}(\mathcal{Y})$ and $f_{j, 1}(\mathcal{Y})$ are defined in (A.7). For simplicity, we choose point mass for $\delta_{j}=\sqrt{2 r \log p / n}$ in (A.7). Then the decision rule can be simplified as

$$
\hat{\theta}_{i}=\mathrm{I}\left\{e_{i}^{T} \tilde{\Sigma}^{-1} \sum_{i=1}^{n_{1}}\left(Y_{i}-\tilde{\delta}\right) \geq \frac{(\beta-\Lambda) \log p}{\sqrt{2 r \log p / n}}+\frac{\omega_{i i} r \log p}{\sqrt{2 r \log p / n}}\right\} .
$$

Since under $H_{0 i}, e_{i}^{T} \tilde{\Sigma}^{-1} \sum_{i=1}^{n_{1}}\left(Y_{i}-\tilde{\delta}\right) \sim \mathrm{N}\left(0, n \omega_{i i}\right)$, under $H_{1 i}, e_{i}^{T} \tilde{\Sigma}^{-1} \sum_{i=1}^{n_{1}}\left(Y_{i}-\right.$ $\tilde{\delta}) \sim \mathrm{N}\left(\sqrt{2 r n \log p} \omega_{i i}, n \omega_{i i}\right)$, we have

$$
\mathrm{E}\left(\hat{\theta}_{i} \mid \theta_{i}=0\right)=\bar{\Phi}\left\{\left(\frac{\beta-\Lambda}{\sqrt{2 \omega_{i i} r}}+\sqrt{\frac{\omega_{i i} r}{2}}\right) \sqrt{\log p}\right\},
$$

and

$$
\mathrm{E}\left(\hat{\theta}_{i} \mid \theta_{i}=1\right)=\bar{\Phi}\left\{\left(\frac{\beta-\Lambda}{\sqrt{2 \omega_{i i} r}}-\sqrt{\frac{\omega_{i i} r}{2}}\right) \sqrt{\log p}\right\},
$$

Since $\beta-\underline{\omega} r<\Lambda<\beta+\underline{\omega} r$,

$$
\mathrm{E}\left(\hat{\theta}_{i} \mid \theta_{i}=0\right)=L_{p} p^{-\frac{\left(\beta-\Lambda+\omega_{i i} r\right)^{2}}{4 \omega_{i i} r}}
$$

and

$$
\mathrm{E}\left(\hat{\theta}_{i} \mid \theta_{i}=1\right)=1-L_{p} p^{-\frac{\left(\omega_{i i} r-\beta+\Lambda\right)^{2}}{4 \omega_{i i} r}} .
$$


First note that in both (A.20) and (A.21), if we choose $\Lambda$ to be $\Lambda_{i i}=$ $\omega_{i i} r+\beta-2 \sqrt{\omega_{i i} r \beta\left(1-\frac{g(\alpha, p)}{\beta}\right)}$ where $g(\alpha, p)=\log \left\{\frac{\alpha}{(1-\alpha)} \sqrt{4 \pi \beta \log p}\right\} \log ^{-1} p$, then the "=" holds in (A.19). To have a universal $\Lambda$ which does not depend on index $i$, we can choose $\Lambda=\underline{\omega} r+\beta-2 \sqrt{\underline{\omega} r \beta\left(1-\frac{g(\alpha, p)}{\beta}\right)}$ such that the right hand is no greater than the left hand of (A.19). Equivalently, this implies that $\mathrm{mFDR} \leq \alpha<1$.

Given $\Lambda$,

$$
\begin{aligned}
\operatorname{mFNR} & =\frac{\mathrm{E}\left\{\sum_{i} \theta_{i}\left(1-\hat{\theta}_{i}\right)\right\}}{\mathrm{E}\left\{\sum_{i}\left(1-\hat{\theta}_{i}\right)\right\}}=\frac{\sum_{i} L_{p} p^{-\beta-\frac{\left(\omega_{i i} r-\beta+\Lambda\right)^{2}}{4 \omega_{i i} r}}}{p\{1+o(1)\}} \\
& \geq \frac{L_{p} p^{1-\beta-\frac{(\bar{\omega} r-\beta+\Lambda)^{2}}{4 \bar{\omega} r}}}{p\{1+o(1)\}} \\
& \geq L_{p} p^{-\beta-\{\sqrt{\bar{\omega} r}-\sqrt{\beta-g(\alpha, p)}\}^{2}} .
\end{aligned}
$$

This completes the proof of Theorem 2 .

A.6. Proof of Theorem 3. Note that the loss function $L\left\{\theta_{j}, \operatorname{sgn}\left(\hat{\delta}_{j}\right)\right\}=$ $\theta_{j}\left\{1-\operatorname{sgn}\left(\hat{\delta}_{j}\right)\right\}+p^{-\Lambda}\left(1-\theta_{j}\right) \operatorname{sgn}\left(\hat{\delta}_{j}\right)$ where $\theta_{j}=0$ if $\delta_{j}=0$ and $\theta_{j}=1$ otherwise, and $\hat{\delta}_{j}$ is estimated to be one of three values from $\left\{-\delta^{\text {data }}, 0, \delta^{\text {date }}\right\}$ by the DATE procedure. Since after the thresholding step, all the coordinates are assigned into either $\mathcal{U}(s)$ or $\mathcal{U}^{c}(s)$, the corresponding risk is

$$
H(\Lambda)=\sum_{j=1}^{p} \mathrm{E}\left(L\left\{\theta_{j}, \operatorname{sgn}\left(\hat{\delta}_{j}\right)\right\}\right)=I+I I,
$$

where $I$ is the risk in the thresholding step and $I I$ is the risk in the excising step, i.e.,

$I=\sum_{j=1}^{p} \mathrm{E}\left(L\left\{\theta_{j}, \operatorname{sgn}\left(\hat{\delta}_{j}\right)\right\} \mathrm{I}\{j \notin \mathcal{U}(s)\}\right), \quad I I=\sum_{j=1}^{p} \mathrm{E}\left(L\left\{\theta_{j}, \operatorname{sgn}\left(\hat{\delta}_{j}\right)\right\} \mathrm{I}\{j \in \mathcal{U}(s)\}\right)$.

For $I$, we know that if $j \notin \mathcal{U}(s)$, the estimated signal $\hat{\delta}_{j}=0$ based on the DATE procedure. By Lemma 3,

$$
I=\sum_{j=1}^{p} \mathrm{P}\left(\hat{T}_{j}<2 s \log p, \delta_{j} \neq 0\right) \leq L_{p} p^{\left.1-\{\underline{\omega} r-\beta+\Lambda)^{2}+4 \underline{\omega} r \beta\right\} /(4 \underline{\omega} r)},
$$


which is not greater than the upper bound of $H(\Lambda)$ except a slowly varying

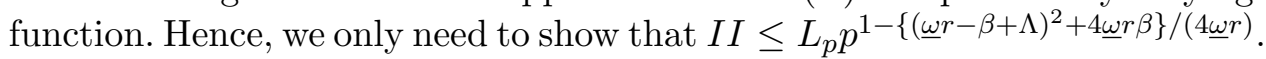

Let event $A_{p}=\{\mathcal{U}(s)$ are split into disconnected clusters of size no more than $K$ with respect to $\left.\left(V_{0}, \Omega^{*}\right)\right\}$. By Lemma $4, \mathrm{P}\left(A_{p}^{c}\right) \leq L_{p} p^{-\frac{(\underline{\omega} r-\beta+\Lambda)^{2}+4 \underline{\omega} r \beta}{4 \underline{\omega} r}}$. Therefore, it is sufficient to show that for all $1 \leq j \leq p$,

$$
\mathrm{E}\left(L\left\{\theta_{j}, \operatorname{sgn}\left(\hat{\delta}_{j}\right)\right\} \mathrm{I}\left\{(j \in \mathcal{U}(s)) \cap A_{p}\right\}\right) \leq L_{p} p^{-\left\{(\underline{\omega} r-\beta+\Lambda)^{2}+4 \underline{\omega} r \beta\right\} /(4 \underline{\omega} r)} .
$$

By Lemma 4, we know that over the event $\{j \in \mathcal{U}(s)\} \cap A_{p}$, there exists a unique component $\mathcal{I}_{0}=\left\{i_{i}, \cdots, i_{m}\right\}$ with size $m \leq K$ satisfying $j \in \mathcal{I}_{0}$. Therefore, it is sufficient to show that for any fixed connected subgroup $\mathcal{I}_{0}$ that contains $j$,

$$
\mathrm{E}\left(L\left\{\theta_{j}, \operatorname{sgn}\left(\hat{\delta}_{j}\right)\right\} \mathrm{I}\left\{\left(j \in \mathcal{I}_{0}\right) \cap A_{p}\right\}\right) \leq L_{p} p^{-\left\{(\underline{\omega} r-\beta+\Lambda)^{2}+4 \underline{\omega} r \beta\right\} /(4 \underline{\omega} r)} .
$$

Recall that $L\left\{\theta_{j}, \operatorname{sgn}\left(\hat{\delta}_{j}\right)\right\}$ consists of the expected false positive and false negative. Accordingly if we define two events

$$
R_{1}=\left\{\theta_{j}=0, \operatorname{sgn}\left(\hat{\delta}_{j}\right) \neq 0, j \in \mathcal{I}_{0}, A_{p}\right\}
$$

and

$$
R_{2}=\left\{\theta_{j}=1, \operatorname{sgn}\left(\hat{\delta}_{j}\right)=0, j \in \mathcal{I}_{0}, A_{p}\right\},
$$

then to show (A.22), we only need to show that

$p^{-\Lambda} \mathrm{P}\left(R_{1}\right) \leq L_{p} p^{-\left\{(\underline{\omega} r-\beta+\Lambda)^{2}+4 \underline{\omega} r \beta\right\} /(4 \underline{\omega} r)}, \mathrm{P}\left(R_{2}\right) \leq L_{p} p^{-\left\{(\underline{\omega} r-\beta+\Lambda)^{2}+4 \underline{\omega} r \beta\right\} /(4 \underline{\omega} r)}$.

Within the component $\mathcal{I}_{0}$, by conducting the DATE procedure, it is possible that some signals are wrongly identified as noise and some noise can be identified as signals. For convenience, we let $B_{n n}$ be the number of true negatives, $B_{n s}$ be the number of false positives, $B_{s n}$ be the number of false negatives, and $B_{s s}$ be the number of true positives. Then, the total number of signals in $\mathcal{I}_{0}$ is $B_{s n}+B_{s s}$. Let the event $G_{p}=\left\{\operatorname{sgn}\left(\hat{\delta}\left(\mathcal{I}_{0}\right) \neq \operatorname{sgn}\left(\delta^{\mathcal{I}_{0}}\right), j \in\right.\right.$ $\left.\mathcal{I}_{0}, A_{p}\right\}$. Since $j \in \mathcal{I}_{0}$, the event $R_{1}$ is contained in $G_{p}$ and the event $R_{2}$ is contained in the event $G_{p} \cap\left\{B_{s n}+B_{s s} \geq 1\right\}$. Therefore, to show (A.23), we only need to show

$$
\begin{aligned}
p^{-\Lambda} \mathrm{P}\left(G_{p}\right) & \leq L_{p} p^{-\left\{(\underline{\omega} r-\beta+\Lambda)^{2}+4 \underline{\omega} r \beta\right\} /(4 \underline{\omega} r)}, \\
\mathrm{P}\left(G_{p} \cap\left\{B_{s n}+B_{s s} \geq 1\right\}\right) & \leq L_{p} p^{-\left\{(\underline{\omega} r-\beta+\Lambda)^{2}+4 \underline{\omega} r \beta\right\} /(4 \underline{\omega} r)} .
\end{aligned}
$$


Let the event $D_{p}=\left\{|| \hat{\Omega}-\Omega \|_{L_{1}} \leq c_{p} M_{p}^{1-q}(\log p / n)^{(1-q) / 2}\right\}$. Since $\mathrm{P}\left(D_{p}^{c}\right)=$ $o\left(p^{-1}\right)$, it is sufficient to show (A.24) over the event $D_{p}$. Moreover, define the event $B_{p}\left(\mathcal{I}_{0}\right)$ through its complement: $B_{p}^{c}\left(\mathcal{I}_{0}\right)=\left\{\right.$ there exist indices $i \notin \mathcal{I}_{0}$ and $j \in \mathcal{I}_{0}$ such that $\left.\delta_{i} \neq 0, \Omega^{*}(i, j) \neq 0\right\}$. Similar to Ji and Jin (2012), we can show that

$$
\mathrm{P}\left(j \in \mathcal{I}_{0}, B_{p}^{c} \cap A_{p}\right) \leq L_{p} p^{-\left\{(\underline{\omega} r-\beta+\Lambda)^{2}+4 \underline{\omega} r \beta\right\} /(4 \underline{\omega} r)} .
$$

Therefore, in order to show (A.24), it is sufficient to show that

$$
\begin{aligned}
p^{-\Lambda} \mathrm{P}\left(G_{p} \cap B_{p} \cap D_{p}\right) & \leq L_{p} p^{-\left\{(\underline{\omega} r-\beta+\Lambda)^{2}+4 \underline{\omega} r \beta\right\} /(4 \underline{\omega} r)}, \\
\mathrm{P}\left(G_{p} \cap B_{p} \cap D_{p} \cap\left\{B_{s n}+B_{s s} \geq 1\right\}\right) & \leq L_{p} p^{-\left\{(\underline{\omega} r-\beta+\Lambda)^{2}+4 \underline{\omega} r \beta\right\} /(4 \underline{\omega} r)} .
\end{aligned}
$$

Most importantly, by Lemma A.4 of Ji and Jin (2012), over the event $\left\{\left(j \in \mathcal{I}_{0}\right) \cap A_{p} \cap B_{p}\right\}$,

$$
\left\|(\Omega \delta)^{\mathcal{I}_{0}}-\Omega^{\mathcal{I}_{0} \mathcal{I}_{0}} \delta^{\mathcal{I}_{0}}\right\|_{L_{1}}=o(\sqrt{\log p / n})
$$

which implies that $(\Omega \delta)^{\mathcal{I}_{0}} \approx \Omega^{\mathcal{I}_{0} \mathcal{I}_{0}} \delta^{\mathcal{I}_{0}}$. This enables us to find $\hat{\delta}\left(\mathcal{I}_{0}\right)$ over the event $G_{p} \cap B_{p} \cap D_{p}$, each components of which has the value taken from $\left\{-\delta^{\text {date }}, 0, \delta^{\text {date }}\right\}$ to minimize

$$
n\left\{\left(\bar{Z}_{1}-\bar{Z}_{2}\right)^{\mathcal{I}_{0}}-A \delta\right\}^{T} A^{-1}\left\{\left(\bar{Z}_{1}-\bar{Z}_{2}\right)^{\mathcal{I}_{0}}-A \delta\right\}+\left(\lambda^{\text {date }}\right)^{2}\|\delta\|_{0},
$$

where $A=\Omega^{\mathcal{I}_{0} \mathcal{I}_{0}}$.

If the event $\left\{G_{p} \cap B_{p} \cap D_{p}\right\}$ happens, then by (A.26),

$$
\begin{aligned}
& n\left\{\left(\bar{Z}_{1}-\bar{Z}_{2}\right)^{\mathcal{I}_{0}}-\Omega^{\mathcal{I}_{0} \mathcal{I}_{0}} \hat{\delta}\left(\mathcal{I}_{0}\right)\right\}^{T}\left(\Omega^{\mathcal{I}_{0} \mathcal{I}_{0}}\right)^{-1}\left\{\left(\bar{Z}_{1}-\bar{Z}_{2}\right)^{\mathcal{I}_{0}}-\Omega^{\mathcal{I}_{0} \mathcal{I}_{0}} \hat{\delta}\left(\mathcal{I}_{0}\right)\right\} \\
+ & \left(\lambda^{\text {date }}\right)^{2}\left\|\hat{\delta}\left(\mathcal{I}_{0}\right)\right\|_{0} \\
\leq & n\left\{\left(\bar{Z}_{1}-\bar{Z}_{2}\right)^{\mathcal{I}_{0}}-\Omega^{\mathcal{I}_{0} \mathcal{I}_{0}} \delta^{*}\left(\mathcal{I}_{0}\right)\right\}^{T}\left(\Omega^{\mathcal{I}_{0} \mathcal{I}_{0}}\right)^{-1}\left\{\left(\bar{Z}_{1}-\bar{Z}_{2}\right)^{\mathcal{I}_{0}}-\Omega^{\mathcal{I}_{0} \mathcal{I}_{0}} \delta^{*}\left(\mathcal{I}_{0}\right)\right\} \\
+ & \left(\lambda^{\text {date }}\right)^{2}\left\|\delta^{*}\left(\mathcal{I}_{0}\right)\right\|_{0},
\end{aligned}
$$

where $\delta^{*}\left(\mathcal{I}_{0}\right)$ is defined to be a vector on $\mathcal{I}_{0}$ each component of which corresponds to the true signals or noise in the sense that for $l \in \mathcal{I}_{0}, \delta_{l}^{*}=0$ if $\delta_{l}=0$ and $\delta_{l}^{*}=\delta^{\text {date }} \operatorname{sgn}(\delta)$ if $\delta_{l} \neq 0$. If we let $d=\left\|\delta^{*}\left(\mathcal{I}_{0}\right)\right\|_{0}-\left\|\hat{\delta}\left(\mathcal{I}_{0}\right)\right\|_{0}=$ $B_{s n}-B_{n s}$, it follows that

$$
\begin{array}{ll}
n\left\{\delta^{*}\left(\mathcal{I}_{0}\right)-\hat{\delta}\left(\mathcal{I}_{0}\right)\right\}^{T}\left(\bar{Z}_{1}-\bar{Z}_{2}\right)^{\mathcal{I}_{0}} & \leq \frac{1}{2}\left(\left(\lambda^{\text {date }}\right)^{2} d+n\left\{\delta^{*}\left(\mathcal{I}_{0}\right)\right\}^{T} \Omega^{\mathcal{I}_{0} \mathcal{I}_{0}} \delta^{*}\left(\mathcal{I}_{0}\right)\right. \\
(\text { A.27) } & \left.-\left\{\hat{\delta}\left(\mathcal{I}_{0}\right)\right\}^{T} \Omega^{\mathcal{I}_{0} \mathcal{I}_{0}} \hat{\delta}\left(\mathcal{I}_{0}\right)\right) .
\end{array}
$$


Since $\sqrt{n}\left\{\left(\bar{Z}_{1}-\bar{Z}_{2}\right)^{\mathcal{I}_{0}}\right\}=\sqrt{n} \Omega^{\mathcal{I}_{0} \mathcal{I}_{0}} \delta^{\mathcal{I}_{0}}+z$ where $z \sim \mathrm{N}\left(0, \Omega^{\mathcal{I}_{0} \mathcal{I}_{0}}\right)$. Then, (A.27) can be written as

$$
\frac{\Delta_{1}^{T} z}{\sqrt{\Delta_{1}^{T} \Omega^{\mathcal{I}_{0} \mathcal{I}_{0}} \Delta_{1}}} \leq-\frac{\sqrt{2 r \log p}}{2 \sqrt{\Delta_{1}^{T} \Omega^{\mathcal{I}_{0} \mathcal{I}_{0}} \Delta_{1}}}\left\{-d \frac{\beta-\Lambda}{r}+2 \Delta_{1}^{T} \Omega^{\mathcal{I}_{0} \mathcal{I}_{0}} \Delta_{2}+\Delta_{1}^{T} \Omega^{\mathcal{I}_{0} \mathcal{I}_{0}} \Delta_{1}\right\},
$$

where

$$
\Delta_{1}=\frac{\sqrt{n}\left\{\left(\delta^{*}\right)^{\mathcal{I}_{0}}-\hat{\delta}\left(\mathcal{I}_{0}\right)\right\}}{\sqrt{2 r \log p}}, \quad \Delta_{2}=\frac{\sqrt{n}\left\{\delta^{\mathcal{I}_{0}}-\left(\delta^{*}\right)^{\mathcal{I}_{0}}\right\}}{\sqrt{2 r \log p}} .
$$

In (A.28), both $\delta^{\mathcal{I}_{0}}$ and $z$ are random. Given $\delta^{\mathcal{I}_{0}}$,

$$
\frac{\Delta_{1}^{T} z}{\sqrt{\Delta_{1}^{T} \Omega^{\mathcal{I}_{0} \mathcal{I}_{0}} \Delta_{1}}} \sim \mathrm{N}(0,1)
$$

Note that if the event $\left\{G_{p} \cap B_{p} \cap D_{p}\right\}$ happens, then the inequality (A.28) holds. Therefore,

$$
\begin{aligned}
\mathrm{P}\left(G_{p} \cap B_{p} \cap D_{p}\right) & \leq \mathrm{P}\left(\frac{\Delta_{1}^{T} z}{\sqrt{\Delta_{1}^{T} \Omega^{\mathcal{I}_{0} \mathcal{I}_{0}} \Delta_{1}}} \leq-\frac{\sqrt{2 r \log p}}{2 \sqrt{\Delta_{1}^{T} \Omega^{\mathcal{I}_{0} \mathcal{I}_{0}} \Delta_{1}}}\left\{-d \frac{\beta-\Lambda}{r}\right.\right. \\
& \left.\left.+2 \Delta_{1}^{T} \Omega^{\mathcal{I}_{0} \mathcal{I}_{0}} \Delta_{2}+\Delta_{1}^{T} \Omega^{\mathcal{I}_{0} \mathcal{I}_{0}} \Delta_{1}\right\}\right) .
\end{aligned}
$$

Next, we evaluate the right hand side of the inequality (A.29) for different values of $B_{s n}+B_{s s}$. To this end, we first notice that the right hand side is bounded by $p^{-\beta\left(B_{s n}+B_{s s}\right)}$, which is the probability of having $B_{s n}+B_{s s}$ signals in $\mathcal{I}_{0}$. Therefore, if $B_{s n}+B_{s s} \geq\left\{(\underline{\omega} r-\beta+\Lambda)^{2}+4 \underline{\omega} r \beta\right\} /(4 \underline{\omega} r \beta)$, from (A.29), it is easy to show that (A.25) is true. Hence, we only need to consider the case where $B_{s n}+B_{s s}<\left\{(\underline{\omega} r-\beta+\Lambda)^{2}+4 \underline{\omega} r \beta\right\} /(4 \underline{\omega} r \beta)$. Note that the value of $B_{n n}$ does not affect the inequality in (A.29). Therefore, we assume $B_{n n}=0$. Also similar to Lemma A.6 of Ji and Jin (2012), it can be shown that $\Delta_{1}^{T} \Omega^{\mathcal{I}_{0} \mathcal{I}_{0}} \Delta_{1} \geq \underline{\omega}$. Moreover, since the support of any signal $\left|\delta_{k}\right|$ is $[\sqrt{2 r \log p / n},(1+\eta) \sqrt{2 r \log p / n}], \Delta_{2} \geq 0$ if $\operatorname{sgn}\left(\delta_{k}\right)=1$ and $\Delta_{2}<0$ otherwise.

- $B_{s n}+B_{s s}=0$;

For this case, we have $d=B_{s n}-B_{n s}=-B_{n s} \leq-1$. Using the fact that $\Delta_{1}^{T} \Omega^{\mathcal{I}_{0} \mathcal{I}_{0}} \Delta_{1} \geq \underline{\omega}$ and $\Delta_{2}=0$, we have

$$
\frac{-d \frac{\beta-\Lambda}{r}+2 \Delta_{1}^{T} \Omega^{\mathcal{I}_{0} \mathcal{I}_{0}} \Delta_{2}+\Delta_{1}^{T} \Omega^{\mathcal{I}_{0} \mathcal{I}_{0}} \Delta_{1}}{2 \sqrt{\Delta_{1}^{T} \Omega^{\mathcal{I}_{0} \mathcal{I}_{0}} \Delta_{1}}} \geq \frac{\frac{\beta-\Lambda}{r}+\Delta_{1}^{T} \Omega^{\mathcal{I}_{0} \mathcal{I}_{0}} \Delta_{1}}{2 \sqrt{\Delta_{1}^{T} \Omega^{\mathcal{I}_{0} \mathcal{I}_{0}} \Delta_{1}}} \geq \frac{\frac{\beta-\Lambda}{r}+\underline{\omega}}{2 \sqrt{\underline{\omega}}} .
$$


Then, from (A.29), by using the fact that $\bar{\Phi}(a) \approx \phi(a) / a$ if $a \rightarrow \infty$, we have

$$
\mathrm{P}\left(G_{p} \cap B_{p} \cap D_{p}\right) \leq \bar{\Phi}\left(-\frac{\frac{\beta-\Lambda}{r}+\underline{\omega}}{2 \sqrt{\underline{\omega}}} \sqrt{2 r \log p}\right) \leq L_{p} p^{-\frac{\underline{(\underline{\omega} r+\beta-\Lambda)^{2}}}{4 \underline{\omega} r}} .
$$

Then, for this case,

$$
\mathrm{P}\left(R_{1}\right) \leq L_{p} p^{-\frac{(\underline{\omega} r+\beta-\Lambda)^{2}}{4 \underline{\omega} r}} .
$$

- $B_{s n}+B_{s s}=1$ but $B_{n s}=0$;

For this case, $\operatorname{since} \operatorname{sgn}\left(\hat{\delta}\left(\mathcal{I}_{0}\right)\right) \neq \operatorname{sgn}\left(\delta^{\mathcal{I}_{0}}\right)$, we must have $B_{s n} \neq 0$. Otherwise, both $B_{s n}=0$ and $B_{n s}=0$ leads to $\operatorname{sgn}\left(\hat{\delta}\left(\mathcal{I}_{0}\right)\right)=\operatorname{sgn}\left(\delta^{\mathcal{I}_{0}}\right)$. As a result, $B_{s n}=1$ and $B_{s s}=0$. It follows that $d=B_{s n}-B_{n s}=1$, $\Delta_{1}^{T} \Omega^{\mathcal{I}_{0} \mathcal{I}_{0}} \Delta_{1} \geq \underline{\omega}$, and $\Delta_{1}^{T} \Omega^{\mathcal{I}_{0} \mathcal{I}_{0}} \Delta_{2} \geq 0$. Then,

$$
\frac{-d \frac{\beta-\Lambda}{r}+2 \Delta_{1}^{T} \Omega^{\mathcal{I}_{0} \mathcal{I}_{0}} \Delta_{2}+\Delta_{1}^{T} \Omega^{\mathcal{I}_{0} \mathcal{I}_{0}} \Delta_{1}}{2 \sqrt{\Delta_{1}^{T} \Omega^{\mathcal{I}_{0} \mathcal{I}_{0}} \Delta_{1}}} \geq \frac{-\frac{\beta-\Lambda}{r}+\Delta_{1}^{T} \Omega^{\mathcal{I}_{0} \mathcal{I}_{0}} \Delta_{1}}{2 \sqrt{\Delta_{1}^{T} \Omega^{\mathcal{I}_{0} \mathcal{I}_{0}} \Delta_{1}}} \geq \frac{-\frac{\beta-\Lambda}{r}+\underline{\omega}}{2 \sqrt{\underline{\omega}}} .
$$

This, together with $B_{s n}+B_{s s}=1$, shows that (A.29) satisfies

$$
\mathrm{P}\left(G_{p} \cap B_{p} \cap D_{p}\right) \leq L_{p} p^{-\beta} p^{-\frac{(\underline{\omega} r-\beta+\Lambda)^{2}}{4 \underline{\omega} r}},
$$

which implies that

$$
\mathrm{P}\left(R_{1}\right) \leq L_{p} p^{-\beta} p^{-\frac{(\underline{\omega} r-\beta+\Lambda)^{2}}{4 \underline{\omega} r}}, \mathrm{P}\left(R_{2}\right) \leq L_{p} p^{-\beta} p^{-\frac{(\underline{\omega} r-\beta+\Lambda)^{2}}{4 \underline{\omega} r}} .
$$

Next, we consider $1 \leq B_{s n}+B_{s s} \leq\left\{(\underline{\omega} r-\beta+\Lambda)^{2}+4 \underline{\omega} r \beta\right\} /(4 \underline{\omega} r \beta)$, $B_{n n}=0$, and when $B_{n s}=0, B_{s n}+B_{s s} \geq 2$. To this end, we apply the Cauchy-Schwartz to get

$$
\left|\Delta_{1}^{T} \Omega^{\mathcal{I}_{0} \mathcal{I}_{0}} \Delta_{2}\right| \leq \sqrt{\Delta_{1}^{T} \Omega^{\mathcal{I}_{0} \mathcal{I}_{0}} \Delta_{1}} \sqrt{\Delta_{2}^{T} \Omega^{\mathcal{I}_{0} \mathcal{I}_{0}} \Delta_{2}}
$$

Using the spectral decomposition, $\Delta_{2}^{T} \Omega^{\mathcal{I}_{0} \mathcal{I}_{0}} \Delta_{2} \leq C_{0}\left\|\Delta_{2}\right\|_{2}^{2}$ where $C_{0}$ is defined in condition (C2). Since the support of signal is $[\sqrt{2 r \log p / n},(1+$

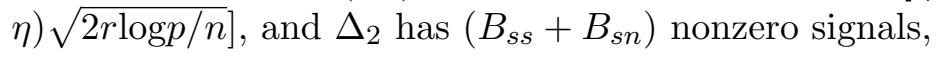

$$
\Delta_{2}^{T} \Omega^{\mathcal{I}_{0} \mathcal{I}_{0}} \Delta_{2} \leq C_{0}\left(B_{s s}+B_{s n}\right) \eta^{2} .
$$

Moreover, with assumption $B_{s n}+B_{s s} \leq\left\{(\underline{\omega} r-\beta+\Lambda)^{2}+4 \underline{\omega} r \beta\right\} /(4 \underline{\omega} r \beta)$, (A.32) can be written as

$$
\left|\Delta_{1}^{T} \Omega^{\mathcal{I}_{0} \mathcal{I}_{0}} \Delta_{2}\right| \leq \sqrt{C} \cdot \sqrt{\Delta_{1}^{T} \Omega^{\mathcal{I}_{0} \mathcal{I}_{0}} \Delta_{1}}
$$


where $C=C_{0} \eta^{2}\left\{(\underline{\omega} r-\beta+\Lambda)^{2}+4 \underline{\omega} r \beta\right\} /(4 \underline{\omega} r \beta)$. Then it follows that $\Delta_{1}^{T} \Omega^{\mathcal{I}_{0} \mathcal{I}_{0}} \Delta_{2} \geq-\sqrt{C} \cdot \sqrt{\Delta_{1}^{T} \Omega^{\mathcal{I}_{0} \mathcal{I}_{0}} \Delta_{1}}$, which implies that

$$
\frac{-d \frac{\beta-\Lambda}{r}+2 \Delta_{1}^{T} \Omega^{\mathcal{I}_{0} \mathcal{I}_{0}} \Delta_{2}+\Delta_{1}^{T} \Omega^{\mathcal{I}_{0} \mathcal{I}_{0}} \Delta_{1}}{2 \sqrt{\Delta_{1}^{T} \Omega^{\mathcal{I}_{0} \mathcal{I}_{0}} \Delta_{1}}} \geq \frac{-d \frac{\beta-\Lambda}{r}+\Delta_{1}^{T} \Omega^{\mathcal{I}_{0} \mathcal{I}_{0}} \Delta_{1}}{2 \sqrt{\Delta_{1}^{T} \Omega^{\mathcal{I}_{0} \mathcal{I}_{0}} \Delta_{1}}}-\sqrt{C} .
$$

Next we consider three different cases: $B_{n s}=B_{s n} \geq 1 ; B_{n s}>B_{s n}$; $B_{n s}<B_{\text {sn }}$.

For the case $B_{n s}=B_{s n} \geq 1$, we have $d=B_{s n}-B_{n s}=0$. Then,

$$
\frac{-d \frac{\beta-\Lambda}{r}+2 \Delta_{1}^{T} \Omega^{\mathcal{I}_{0} \mathcal{I}_{0}} \Delta_{2}+\Delta_{1}^{T} \Omega^{\mathcal{I}_{0} \mathcal{I}_{0}} \Delta_{1}}{2 \sqrt{\Delta_{1}^{T} \Omega^{\mathcal{I}_{0} \mathcal{I}_{0}} \Delta_{1}}} \geq \frac{\sqrt{\Delta_{1}^{T} \Omega^{\mathcal{I}_{0} \mathcal{I}_{0}} \Delta_{1}}}{2}-\sqrt{C} .
$$

Recall that $\Delta_{1}^{T} \Omega^{\mathcal{I}_{0} \mathcal{I}_{0}} \Delta_{1} \geq \underline{\omega}$ and $\eta \leq \frac{\beta-\Lambda}{\sqrt{C_{0} r}} \frac{\sqrt{\beta r}}{\sqrt{(\underline{\omega} r-\beta+\Lambda)^{2}+4 \underline{\omega} r \beta}}$. Then, $\sqrt{C} \leq(\beta-\Lambda) /(2 \sqrt{\underline{\omega}} r)$, and

$$
\mathrm{P}\left(G_{p} \cap B_{p} \cap D_{p}\right) \leq L_{p} p^{-\beta} p^{-\frac{(\underline{\omega} r-\beta+\Lambda)^{2}}{4 \underline{\omega} r}} .
$$

For the case $B_{n s}>B_{s n}, d=B_{s n}-B_{n s} \leq-1$. Since $\eta \leq \frac{\beta-\Lambda}{\sqrt{C_{0} r}} \frac{\sqrt{\beta r}}{\sqrt{(\underline{\omega r}-\beta+\Lambda)^{2}+4 \underline{\omega r} \beta}}$, we have $\sqrt{C} \leq(\beta-\Lambda) /(\sqrt{\underline{\omega}} r)$, and

$$
\begin{aligned}
\frac{-d \frac{\beta-\Lambda}{r}+2 \Delta_{1}^{T} \Omega^{\mathcal{I}_{0} \mathcal{I}_{0}} \Delta_{2}+\Delta_{1}^{T} \Omega^{\mathcal{I}_{0} \mathcal{I}_{0}} \Delta_{1}}{2 \sqrt{\Delta_{1}^{T} \Omega^{\mathcal{I}_{0} \mathcal{I}_{0}} \Delta_{1}}} & \geq \frac{\frac{\beta-\Lambda}{r}+\Delta_{1}^{T} \Omega^{\mathcal{I}_{0} \mathcal{I}_{0}} \Delta_{1}}{2 \sqrt{\Delta_{1}^{T} \Omega^{\mathcal{I}_{0} \mathcal{I}_{0}} \Delta_{1}}}-\sqrt{C} \\
& \geq \frac{1}{2}\left(\frac{\beta-\Lambda}{\sqrt{\underline{\omega}} r}+\sqrt{\underline{\omega}}\right)-\sqrt{C} \\
& \geq \frac{1}{2}\left(\sqrt{\underline{\omega}}-\frac{\beta-\Lambda}{\sqrt{\underline{\omega}} r}\right) .
\end{aligned}
$$

Since $B_{s n}+B_{s s} \geq 1$, then

$$
\mathrm{P}\left(G_{p} \cap B_{p} \cap D_{p}\right) \leq L_{p} p^{-\beta} p^{-\frac{(\underline{\omega} r-\beta+\Lambda)^{2}}{4 \underline{\omega} r}} .
$$

For the case $B_{n s}<B_{s n}$, we have either $B_{n s}=0$ or $B_{n s} \geq 1$. If $B_{n s}=0, B_{s n}+B_{s s} \geq 2$ as we have required. If $B_{n s} \geq 1$, we also have $B_{s n}+B_{s s} \geq 2$ due to the fact that $B_{s n}>B_{n s}$. Since $\beta+(\underline{\omega} r-\beta+$ $\Lambda)^{2} /(4 \underline{\omega} r)=\Lambda+(\underline{\omega} r+\beta-\Lambda)^{2} /(4 \underline{\omega} r)$ and $\beta-\Lambda<\underline{\omega} r$, then

$$
\beta+(\underline{\omega} r-\beta+\Lambda)^{2} /(4 \underline{\omega} r) \leq \Lambda+\underline{\omega} r .
$$


Then using the fact that $\beta\left(B_{s n}+B_{s s}\right) \geq 2 \beta$ and by assuming $\beta>1 / 2$ and $\underline{\omega} r<(\sqrt{1-\Lambda}+\sqrt{1-\beta})^{2}$, we can derive the following inequality

$$
\beta\left(B_{s n}+B_{s s}\right) \geq \beta+\frac{(\underline{\omega} r-\beta+\Lambda)^{2}}{4 \underline{\omega} r} .
$$

Therefore, for three different cases: $B_{n s}=B_{s n} \geq 1 ; B_{n s}>B_{s n} ; B_{n s}<$ $B_{s n}$, we have

$$
\mathrm{P}\left(G_{p} \cap B_{p} \cap D_{p}\right) \leq L_{p} p^{-\beta} p^{-\frac{(\underline{\omega} r-\beta+\Lambda)^{2}}{4 \underline{\omega} r}},
$$

which implies that

$$
\mathrm{P}\left(R_{1}\right) \leq L_{p} p^{-\beta} p^{-\frac{(\underline{\omega} r-\beta+\Lambda)^{2}}{4 \underline{\omega} r}}, \mathrm{P}\left(R_{2}\right) \leq L_{p} p^{-\beta} p^{-\frac{(\underline{\omega} r-\beta+\Lambda)^{2}}{4 \underline{\omega} r}} .
$$

In summary, from (A.30), (A.31) and (A.33), we know that if $B_{s n}+B_{s s}=$ $0, \mathrm{P}\left(R_{1}\right) \leq L_{p} p^{-\frac{(\underline{\omega} r+\beta-\Lambda)^{2}}{4 \underline{\omega} r}}$. And if $B_{s n}+B_{s s} \geq 1, \mathrm{P}\left(R_{1}\right) \leq L_{p} p^{-\beta} p^{-\frac{(\underline{\omega} r-\beta+\Lambda)^{2}}{4 \underline{\omega} r}}$. Since

$$
\frac{(\underline{\omega} r+\beta-\Lambda)^{2}}{4 \underline{\omega} r} \leq \beta+\frac{(\underline{\omega} r-\beta+\Lambda)^{2}}{4 \underline{\omega} r},
$$

we have $\mathrm{P}\left(R_{1}\right) \leq L_{p} p^{-\frac{(\underline{\omega} r+\beta-\Lambda)^{2}}{4 \underline{\omega} r}}$. Similarly, we have $\mathrm{P}\left(R_{2}\right) \leq L_{p} p^{-\beta} p^{-\frac{(\underline{\omega} r-\beta+\Lambda)^{2}}{4 \underline{\omega} r}}$. Then,

$$
p^{-\Lambda} \mathrm{P}\left(R_{1}\right)+\mathrm{P}\left(R_{2}\right) \leq L_{p} p^{-\beta} p^{-\frac{(\underline{\omega} r-\beta+\Lambda)^{2}}{4 \underline{\omega} r}},
$$

which shows that (A.23) is true. This completes the proof of Theorem 3.

A.7. Proof of Theorem 4. Let $\theta_{i}=0$ if $\delta_{i}=0$ and $\theta_{i}=1$ otherwise, and $\hat{\delta}_{i}$ is the corresponding estimate by the DATE procedure. Recall that the marginal false discovery rate is defined as

$$
\operatorname{mFDR}=\frac{\sum_{i} \mathrm{P}\left(\theta_{i}=0, \operatorname{sgn}\left(\hat{\delta}_{i}\right) \neq 0\right)}{\sum_{i} \mathrm{P}\left(\theta_{i}=0, \operatorname{sgn}\left(\hat{\delta}_{i}\right) \neq 0\right)+\sum_{i} \mathrm{P}\left(\theta_{i}=1, \operatorname{sgn}\left(\hat{\delta}_{i}\right) \neq 0\right)} .
$$

Similar to the proof of Theorem 3.2 in Ji and Zhao (2014), we can use (A.29) in the proof of Theorem 3 to derive the following

$$
\begin{aligned}
\mathrm{P}\left\{\theta_{i} \neq \operatorname{sgn}\left(\hat{\delta}_{i}\right)\right\} & \leq p^{-\beta\left(B_{s n}+B_{s s}\right)} \\
(\mathrm{A} .34) & \times \bar{\Phi}\left(\frac{-d \frac{\beta-\Lambda-\frac{r}{2 \log p}}{r}+2 \Delta_{1}^{T} \Omega^{\mathcal{I}_{0} \mathcal{I}_{0}} \Delta_{2}+\Delta_{1}^{T} \Omega^{\mathcal{I}_{0} \mathcal{I}_{0}} \Delta_{1}}{2 \sqrt{\Delta_{1}^{T} \Omega^{\mathcal{I}_{0} \mathcal{I}_{0}} \Delta_{1}}} \sqrt{2 r \log p}\right) .
\end{aligned}
$$


First, note that

$$
\begin{aligned}
\mathrm{P}\left(\theta_{i}=1, \operatorname{sgn}\left(\hat{\delta}_{i}\right) \neq 0\right) & =\mathrm{P}\left(\theta_{i}=1\right)-\mathrm{P}\left(\theta_{i}=1, \operatorname{sgn}\left(\hat{\delta}_{i}\right)=0\right) \\
& =p^{-\beta}-\mathrm{P}\left(\theta_{i}=1, \operatorname{sgn}\left(\hat{\delta}_{i}\right)=0\right) .
\end{aligned}
$$

Following the similar derivations for Theorem 3, from (A.34), we can show that

$$
\mathrm{P}\left(\theta_{i}=1, \operatorname{sgn}\left(\hat{\delta}_{i}\right)=0\right) \leq p^{-\beta} \bar{\Phi}\left(\frac{(-\beta+\Lambda+\underline{\omega} r) \log p+\Upsilon / 2}{\sqrt{2 \underline{\omega} r \log p}}\right),
$$

where $\Upsilon$ is defined in Theorem 4. Then,

$$
\mathrm{P}\left(\theta_{i}=1, \operatorname{sgn}\left(\hat{\delta}_{i}\right) \neq 0\right) \geq p^{-\beta} \bar{\Phi}\left(\frac{(\beta-\Lambda-\underline{\omega} r) \log p-\Upsilon / 2}{\sqrt{2 \underline{\omega} r \log p}}\right) .
$$

Similarly,

$$
\begin{aligned}
\left.\mathrm{P}\left(\theta_{i}=0, \operatorname{sgn}\left(\hat{\delta}_{i}\right) \neq 0\right)\right) & \left.\left.=\mathrm{P}\left(\theta_{i}=0, \operatorname{sgn}\left(\hat{\delta}_{i}\right)=1\right)\right)+\mathrm{P}\left(\theta_{i}=0, \operatorname{sgn}\left(\hat{\delta}_{i}\right)=-1\right)\right) \\
& \leq 2\left(1-p^{-\beta}\right) \bar{\Phi}\left(\frac{(\beta-\Lambda+\underline{\omega} r) \log p-\Upsilon / 2}{\sqrt{2 \underline{\omega} r \log p}}\right) .
\end{aligned}
$$

To require mFDR to be controlled at $\alpha$, we need to find $\Upsilon$ such that

$$
\alpha \geq \frac{2\left(p-p^{1-\beta}\right) \bar{\Phi}\left(\frac{(\beta-\Lambda+\underline{\omega} r) \log p-\Upsilon / 2}{\sqrt{2 \underline{\omega} r \log p}}\right)}{2\left(p-p^{1-\beta}\right) \bar{\Phi}\left(\frac{(\beta-\Lambda+\underline{\omega} r) \log p-\Upsilon / 2}{\sqrt{2 \underline{\omega} r \log p}}\right)+p^{1-\beta} \bar{\Phi}\left(\frac{(\beta-\Lambda-\underline{\omega} r) \log p-\Upsilon / 2}{\sqrt{2 \underline{\omega} r \log p}}\right)} .
$$

When $p \rightarrow \infty$, (A.35) can be solved asymptotically. If we assume $\Upsilon=$ $o(\log p)$, then by the fact that $\underline{\omega} r>\beta-\Lambda$,

$$
\bar{\Phi}\left(\frac{(\beta-\Lambda-\underline{\omega} r) \log p-\Upsilon / 2}{\sqrt{2 \underline{\omega} r \log p}}\right) \rightarrow 1 .
$$

Then using the fact that $\bar{\Phi}(a) \approx \phi(a) / a$ for $a \rightarrow \infty$, we can solve (A.35) by choosing

$$
\Upsilon=\frac{4 \underline{\omega} r}{\underline{\omega} r+\beta-\Lambda}\left(\frac{1}{2} \log \log p+\log \left\{\frac{\alpha \sqrt{\pi}(\underline{\omega} r+\beta-\Lambda)}{2 \sqrt{\underline{\omega} r}(1-\alpha)}\right\}\right)
$$

such that

$$
\begin{aligned}
\operatorname{mFDR} & \leq \frac{\frac{\alpha}{1-\alpha} p^{-\frac{(\underline{\omega} r+\beta-\Lambda)^{2}}{4 \underline{\omega} r}}}{\frac{\alpha}{1-\alpha} p^{-\frac{(\underline{\omega} r+\beta-\Lambda)^{2}}{4 \underline{\omega} r}}+p^{-\beta}\{1+o(1)\}} \\
& \leq \alpha\{1+o(1)\},
\end{aligned}
$$


where we have used the result that $\Lambda=(\sqrt{\underline{\omega} r}-\sqrt{\beta})^{2}$.

Similarly, the marginal false non-discovery rate

$$
\begin{aligned}
\operatorname{mFNR} & =\frac{\sum_{i} \mathrm{P}\left(\theta_{i}=1, \operatorname{sgn}\left(\hat{\delta}_{i}\right)=0\right)}{\sum_{i} \mathrm{P}\left(\theta_{i}=1, \operatorname{sgn}\left(\hat{\delta}_{i}\right)=0\right)+\sum_{i} \mathrm{P}\left(\theta_{i}=0, \operatorname{sgn}\left(\hat{\delta}_{i}\right)=0\right)} \\
& \leq L_{p} p^{-\beta-(\sqrt{\underline{\omega} r}-\sqrt{\beta})^{2}} .
\end{aligned}
$$

This completes the proof of Theorem 4 .

A.8. Proof of Theorem 5. Note that the optimal rate does not change if we add a slowly varying logarithm function to the tuning parameters in Theorem 4 by carefully reviewing its proof. Therefore, if we can show that there exists a constant $C$ such that

$$
\begin{aligned}
& \mathrm{P}\left(|\hat{\beta}-\beta|>(\log p)^{-C}\right)=o\left(p^{-1}\right), \\
& \mathrm{P}\left(|\hat{r}-r|>(\log p)^{-C}\right)=o\left(p^{-1}\right),
\end{aligned}
$$

and

$$
\mathrm{P}\left(|\underline{\hat{\omega}}-\underline{\omega}|>(\log p)^{-C}\right)=o\left(p^{-1}\right),
$$

where $\hat{\beta}, \hat{r}$ and $\underline{\hat{\omega}}$ are defined in (4.6) in the main paper [Li and Zhong (2015)], then Theorem 5 can be proved.

Let's prove (A.36) or equivalently, we need to show that

$$
\mathrm{P}\left(\left|\frac{1}{p^{1-\beta}} \sum_{k=1}^{p} \mathrm{I}\left(\hat{T}_{k}>2 q \log p\right)-1\right|>L_{p} p^{-C}\right)=o\left(p^{-1}\right) .
$$

To this end, we first notice that

$$
\sum_{k=1}^{p} \mathrm{I}\left(\hat{T}_{k}>2 q \log p\right)=\sum_{k=1}^{p} \mathrm{I}\left(\hat{T}_{k}>2 q \log p\right) \mathrm{I}\left(\delta_{k}=0\right)+\sum_{k=1}^{p} \mathrm{I}\left(\hat{T}_{k}>2 q \log p\right) \mathrm{I}\left(\delta_{k} \neq 0\right) .
$$

Then,

$$
\begin{aligned}
& \mathrm{P}\left(\left|\frac{1}{p^{1-\beta}} \sum_{k=1}^{p} \mathrm{I}\left(\hat{T}_{k}>2 q \log p\right)-1\right|>L_{p} p^{-C}\right) \\
\leq & \mathrm{P}\left(\left|\frac{1}{p^{1-\beta}} \sum_{k=1}^{p} \mathrm{I}\left(\hat{T}_{k}>2 q \log p\right) \mathrm{I}\left(\delta_{k}=0\right)\right|>L_{p} p^{-C}\right) \\
+ & \mathrm{P}\left(\left|\frac{1}{p^{1-\beta}} \sum_{k=1}^{p} \mathrm{I}\left(\hat{T}_{k}>2 q \log p\right) \mathrm{I}\left(\delta_{k} \neq 0\right)-1\right|>L_{p} p^{-C}\right) .
\end{aligned}
$$


Using Chebyshev's inequality and the result in Lemma A.7 of Ji and Jin (2012), we have

$$
\begin{aligned}
& \mathrm{P}\left(\left|\frac{1}{p^{1-\beta}} \sum_{k=1}^{p} \mathrm{I}\left(\hat{T}_{k}>2 q \log p\right) \mathrm{I}\left(\delta_{k}=0\right)\right|>L_{p} p^{-C}\right) \\
\leq & \frac{\mathrm{E}\left(\left\{\sum_{k=1}^{p} \mathrm{I}\left(\hat{T}_{k}>2 q \log p\right) \mathrm{I}\left(\delta_{k}=0\right)\right\}^{m}\right)}{p^{m-m \beta} L_{p}^{m} p^{-m C}} \\
\leq & \frac{L_{p} p^{m} p^{-m q}}{p^{m-m \beta-m C}} \\
= & L_{p} p^{-m(q-\beta+C)}
\end{aligned}
$$

Then we can choose $m$ large enough to have $p^{-m(q-\beta+C)}=o\left(p^{-1}\right)$ since $q>$ $\beta$. Based on similar derivations, (A.37) and (A.38) can be shown accordingly. This completes the proof of Theorem 5 .

\section{References.}

CAI, T., LiU, W. and Luo, X. (2011). A constrained $l_{1}$ minimization approach to sparse precision matrix estimation. J. Amer. Statist. Assoc. 106 594-607.

Frieze, A. and Molloy, M. (1999). Splitting an expander graph. J. Algorithms 33 166172.

Ji, P. and Jin, J. (2012). UPS delivers optimal phase diagram in high-dimensional variable selection. Ann. Statist. 40 73-103.

Ji, P. and ZнаO, Z. (2014). Rate optimal multiple testing procedure in high-dimensional regression. Technical report.

LI, J. and Zhong, P. (2015). A rate optimal procedure for recovering sparse differences between high-dimensional means under dependence. Technical report.

Sun, W. and CAI, T. (2007). Oracle and adaptive compound decision rules for false discovery rate control. J. Amer. Statist. Assoc. 102 901-912.

XIE, J., CAI, T. and Li, H. (2011). Sample size and power analysis for sparse signal recovery in genome-wide association studies. Biometrika 98 273-290.

Department of Mathematical Sciences

Kent State University

Kent, Оhio 44242

USA

E-MAIL: junli@math.kent.edu
Department of Statistics and Probability Michigan State University

EAST LANSing, Michigan 48823

USA

E-MAIL: pszhong@stt.msu.edu 